\title{
Tuning of Heat Transfer Rate of Cobalt Manganese Ferrite Based Magnetic Fluids in Varying Magnetic Field
}

\author{
Margabandhu MARIMUTHU ${ }^{1}$ *, Sendhilnathan SECHASSALOM ${ }^{2}$, \\ Sirikanjana THONGMEE ${ }^{3}$
}

\author{
${ }^{1}$ Anna University Chennai: University College of Engineering-Ariyalur, Department of Physics, Ariyalur-621704, India \\ ${ }^{2}$ Anna University Chennai: University College of Engineering-Pattukkottai, Department of Physics, Rajamadam, India \\ ${ }^{3}$ Department of Physics, Kasetsart University, Bangkok, 10900, Thailand \\ crossref http://dx.doi.org/10.5755/j01.ms.23.3.16662
}

Received 03 November 2016; accepted 12 January 2017

\begin{abstract}
Magnetic fluids are the colloidal solutions containing suspended magnetic nanoparticles in carrier fluids. The present work analyzed the heat transfer characteristics of de-ionized water and transformer oil (base fluids) based cobalt manganese ferrite $\left(\mathrm{Co}_{1-\mathrm{x}} \mathrm{Mn}_{\mathrm{x}} \mathrm{Fe}_{2} \mathrm{O}_{4}\right.$ coated with oleic acid synthesized via co-precipitation technique) magnetic fluids in varying magnetic field. Experimental investigations were carried out to analyze the heat transfer property of synthesized magnetic fluids (MNF) in varying magnetic field applied in perpendicular direction to the thermal gradient of magnetic fluids. The experimental results indicate that the magnetic fluids show enhancement in heat transfer rate than carrier fluids in absence of magnetic field and it shows decrement in heat transfer rate in presence of varying magnetic field. Thus, the results reveal that the heat transfer characteristics of cobalt manganese ferrite based magnetic fluids was tunable by controlling the direction and influence of magnetic field strength. This tunable heat transfer property of cobalt manganese ferrite based magnetic fluids could be applicable in heat transport phenomena of transformers and in microelectronic devices.

Keywords: ferrites, magnetic nanofluids, base fluids, heat transfer rate, varying magnetic field.
\end{abstract}

\section{INTRODUCTION}

Effective heat transport phenomena is one of the most essential challenge faced by many industries producing microelectronic devices, engine coolants, automobile parts and smart cooling devices. The conventional heat transfer fluids used as coolants are not suffice for rapid heat transportation in advanced microelectronic and in engine cooling devices to meet out the heat transfer process. Therefore, it is an immediate need to identify new coolants to attain ultra-high performance cooling [1]. Fluids possessing micron-sized and nano-sized metallic particles exhibit superior properties in cooling process rather than conventional heat transfer fluids. Specifically, the heat transfer in liquids with metallic particles is much greater than that of non metallic particles dispersed liquids [2]. For example, the heat protruding in copper particles dispersed water is nearly 700 times more than that of normal water at room temperature and 3000 times greater than engine oil. Hence, fluids containing dispersed metallic particles show predominant enhanced heat transfer rather than the traditional heat conduction fluids [3]. Nanofluids that are a new category of fluids consist of solid particles in the size of around $1-100 \mathrm{~nm}$ suspended in base fluids. They exhibit peculiar properties relative than conventional heat transfer fluids. The reasons are: 1) the ratio between surface to volume is about 1000 times greater for particles possessing $10 \mathrm{~nm}$ diameter rather than particles with $10 \mu \mathrm{m}$ diameter; 2 ) the dispersed nanoparticles promotes the heat transfer of the fluid; 3) the mutual interaction among the particles, the fluid and the fluid passage surface becomes improved; 4) the nanoparticles suspension flattens the transverse temperature gradient of the fluid. Thus, drastic increment in heat transfer is obtained as result of particle size decrement in suspended fluid $[4,5]$. Xie et al. [6] reported in his paper about the thermal conductivity analysis of nanosized $\mathrm{SiC}$ suspensions in basefluid. His report shows that the thermal conductivities of $\mathrm{SiC}$ suspensions show linear enhancement with the volume fraction of suspended particles for different systems, it is independent of the base fluids. Effective thermal conductivity of fluid mixtures possessing ethylene glycol, $\mathrm{Al}_{2} \mathrm{O}_{3}, \mathrm{CuO}$ dispersed in water, vacuum pump fluid and engine oil have been delineated by Wang et al. [7]. His experimental results show enhanced thermal conductivity in nano-fluid mixtures than the base fluids. Magnetic nanofluids (MNF) are the colloidal suspensions possess magnetic nanoparticles in basefluids. In magnetic nanofluids, the parameters such as volume fraction of magnetic nanoparticles, particle size/particle size distribution, chemical composition, surfactant and temperature determines the heat transfer property. The strength and direction of magnetic field on magnetic nanofluids (MNF) shows remarkable effects on rate of heat conduction and on viscosity of magnetic nanofluids [8]. Hence, the main focus of the present work is to synthesize oleic acid coated cobalt manganese ferrites and to investigate the heat transfer characteristics of de-ionized water and transformer oil (base fluids) based cobalt manganese ferrite magnetic fluids in varying magnetic field.

\section{EXPERIMENTAL DETAILS}

\subsection{Magnetic fluid synthesis}

Cobalt manganese ferrite $\left(\mathrm{Co}_{1-\mathrm{x}} \mathrm{Mn}_{\mathrm{x}} \mathrm{Fe}_{2} \mathrm{O}_{4}\right)$ nanoparticles coated with oleic acid was prepared by adding

\footnotetext{
* Corresponding author. Tel.: +918098144509.

E-mail address: mmargabandhu@yahoo.co.in (M.Margabandhu)
} 
homogenously combined cobalt chloride, manganese chloride and ferric chloride solutions $\left(\mathrm{CoCl}_{2}, \mathrm{MnCl}_{2}\right.$ and $\mathrm{FeCl}_{3}$ ) in alkaline medium $\mathrm{NaOH}$. The molar ratio 1:2 was strictly maintained among added salts with alkaline solution. In synthesis process, the combination of precursors was simultaneously stirred and heated for $1 \mathrm{~h}$ at $85^{\circ} \mathrm{C}$. After $1 \mathrm{~h}$ duration the mixture was added with oleic acid and intended to mechanical stirring for an hour. Finally, the mixture was uninterrupted for few hours to settle down. The settled down particles was drained and often washed with demineralized water to acquire $\mathrm{pH}$ of around 7. At last, acetone was added with the obtained precipitate to remove unnecessary constituents and dried normally. Thus, dried cobalt manganese ferrite samples coated with oleic acid was obtained $[9,10]$. Cobalt manganese ferrite based magnetic fluids were prepared by dispersing $1.25 \%$ volume fraction of cobalt manganese ferrite nanopowders $\left(\mathrm{Co}_{1-\mathrm{x}} \mathrm{Mn}_{\mathrm{x}} \mathrm{Fe}_{2} \mathrm{O}_{4}\right)$ of different molar ratio in base fluids (deionized water and transformer oil). The volume fraction of dispersed particles was estimated by using the formula [11]:

$$
\phi=\frac{\omega_{P} \times \rho_{f}}{\left(\omega_{P} \times \rho_{f}\right)+\rho_{P}(1-\phi)},
$$

where $\omega_{p}$ is the weight particle fraction, $\rho_{p}$ and $\rho_{f}$ are the densities of dispersed phase and base fluid. The solutions were subjected to vigorous stirring for $2 \mathrm{hrs}$ to obtain stable solution. In order to avoid agglomeration of magnetic nanoparticles and to obtain stable magnetic nanofluids, the particle size should be with respect to equation [12]:

$d \leq\left(\frac{144 k_{B} T}{\pi \mu_{0} M^{2}}\right)^{\frac{1}{3}}$,

where $k_{B}, T, M$ are Boltzmann constant, temperature and magnetization respectively. The above said condition will be satisfied only when the particles are in nanometre range. In our case, the suspended particles remain stable, since suspension without agglomeration. It may satisfy the above equation condition. Also, the particles coated with oleic acid may prevent agglomeration and provide stability to the solution. In our case, a carefully governed agglomeration issue finally provides stable cobalt manganese ferrite based magnetic fluids [13].

\subsection{Experimental arrangement for heat transfer analysis}

The experimental set up for analysing the heat transfer rate of cobalt manganese ferrite based magnetic fluids $\left(\mathrm{Co}_{1}\right.$ $\left.{ }_{x} \mathrm{Mn}_{\mathrm{x}} \mathrm{Fe}_{2} \mathrm{O}_{4}\right)$ in varying magnetic field applied in a perpendicular direction in accordance with the temperature gradient is schematically shown in Fig. 1.

The arrangement consists of $500 \mathrm{ml}$ glass beakers, an electromagnet set up, thermometer and heater. The electromagnet was designed in such a way to hold the $500 \mathrm{ml}$ glass beakers with in it. An AC power supply of $50 \mathrm{~Hz}$ frequency was connected to electromagnet through rheostat to provide varying current.

Since, the synthesized magnetic particles are coated with oleic acid, it will not make contact with the surfactant layer. Hence, the applied varying magnetic field will be strong enough to overcome the surfactant layer.

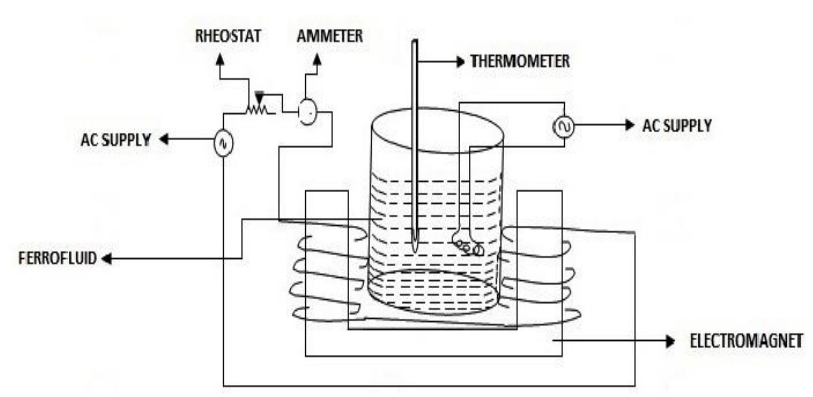

Fig. 1. Schematic representation for the measurement of heat transfer rate of magnetic fluids in varying magnetic field applied outside the beaker

By considering the thickness of the surfactant into account, the interparticle interaction can be calculated as $[14,15]$ :

$\lambda^{*}=\frac{\mu_{0} M_{s p}{ }^{2} V}{24 k_{B} T}\left(\frac{d}{d+2 s}\right)^{3}$,

where $M_{s p}$ is the spontaneous magnetization of the magnetic material of the particles, $d$ is the diameter of the particles, $s$ is the surfactant layer thickness, $k_{B}$ is the Boltzmann constant and $T$ is the temperature respectively. For $\lambda^{*}<1$, there will be no interparticle interaction in magnetic field due to dominant heat energy. For $\lambda^{*}>1$, the heat energy domination will be more than the results in the structural formation in magnetic fields. Magnetic fluids (deionized water and transformer oil) with $1.25 \%$ volume fraction of cobalt manganese ferrite samples $\left(\mathrm{Co}_{1-\mathrm{x}} \mathrm{Mn}_{\mathrm{x}} \mathrm{Fe}_{2} \mathrm{O}_{4}\right)$ of various molar ratio were chosen for heat transfer rate analysis. A heater connected to $\mathrm{AC}$ power supply of $50 \mathrm{~Hz}$ frequency was used to heat magnetic fluids and a thermometer was used to measure the heat transfer rate with respect to time. In this experimental arrangement, the designed electromagnet was used to produce varying magnetic field for the appropriate applied current values. The chosen current values are 1.8 A and 2.7 A respectively. The varying magnetic field was applied outside the glass beakers, in which the direction of the magnetic field would be perpendicular to the direction of thermal gradient. The heat transfer rate of synthesized cobalt manganese ferrite based $\left(\mathrm{Co}_{1-\mathrm{x}} \mathrm{Mn}_{\mathrm{x}} \mathrm{Fe}_{2} \mathrm{O}_{4}\right)$ magnetic fluids of various molar ratio with respected to time was carefully noted and analyzed systematically.

\subsection{Characterization}

X-ray diffraction patterns of prepared powdered samples were measured with X-ray powder diffractometer using $\mathrm{Cu} \mathrm{K}_{\propto}$ radiation in the diffraction range $10^{\circ}$ to $80^{\circ}$ and by using $\lambda=1.5406 \AA$ at room temperature (X' PERT PRO). The results were obtained with step size and scan step time of about $0.05^{\circ}$ and $10 \mathrm{~s}$. Particle morphology was examined using Transmission Electron Microscope (TEM) (JEOL JEM 2100). 


\section{RESULTS AND DISCUSSION}

\subsection{Structure analysis}

All the identified diffraction peaks of maximum intensity at (311) plane confirm the single phase face centered cubic spinel structure of cobalt manganese ferrite powdered samples. The variation in average crystallite size is shown in Table 1 .

Table 1. Average crystallite size (DXRD)(nm) of cobalt manganese ferrite samples

\begin{tabular}{|c|c|}
\hline Samples & Average crystallite size, $\mathrm{nm}$ \\
\hline $\mathrm{CoFe}_{2} \mathrm{O}_{4}$ & 31.95 \\
\hline $\mathrm{Co}_{0.7} \mathrm{Mn}_{0.3} \mathrm{Fe}_{2} \mathrm{O}_{4}$ & 25.68 \\
\hline $\mathrm{Co}_{0.5} \mathrm{Mn}_{0.5} \mathrm{Fe}_{2} \mathrm{O}_{4}$ & 22.73 \\
\hline $\mathrm{Co}_{0.3} \mathrm{Mn}_{0.7} \mathrm{Fe}_{2} \mathrm{O}_{4}$ & 15.85 \\
\hline $\mathrm{MnFe}_{2} \mathrm{O}_{4}$ & 7.53 \\
\hline
\end{tabular}

The lattice constant of the synthesized samples increases from $8.385 \AA$ to $8.495 \AA$ because of the replacement of $\mathrm{Mn}^{2+}$ ions in the location of $\mathrm{Co}^{2+}$ ions whose atomic radii is more than that of $\mathrm{Co}^{2+}$ ions. The average crystallite size of cobalt manganese ferrite $\left(\mathrm{Co}_{1-\mathrm{x}} \mathrm{Mn}_{\mathrm{x}} \mathrm{Fe}_{2} \mathrm{O}_{4}\right)$ powder samples was found to exist in between $31.95 \mathrm{~nm}$ to $7.53 \mathrm{~nm}$. It was estimated by using Debye Scherrer's formula $[9,16]$ :

$D=\frac{0.89 \lambda}{\beta \cos \theta}$,

where $\lambda$ represents $\mathrm{X}$-ray wavelength, $\beta$ is the breath of the obtained maximum reflection plane in half intensity maximum (FWHM represented in radians), $\theta$ is the Bragg's diffraction angle and $D$ indicates the average crystallite size [17].

\subsection{Morphology analysis}

The TEM micrograph of prepared cobalt manganese ferrite nanoparticles is shown in Fig. 2.
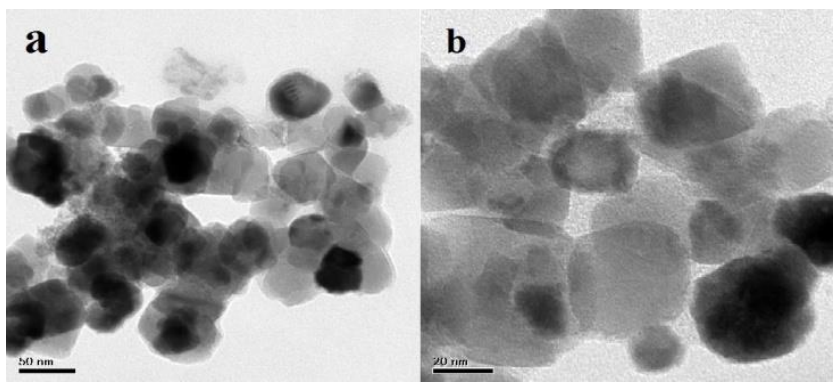

Fig. 2. TEM micrograph: $\mathrm{a}-\mathrm{CoFe}_{2} \mathrm{O}_{4} ; \mathrm{b}-\mathrm{Co}_{0.5} \mathrm{Mn}_{0.5} \mathrm{Fe}_{2} \mathrm{O}_{4}$

The image shows the morphology and homogenous chemical composition of synthesized particles. The image reveals the agglomerated nature of the particles which was due to magnetic interaction among the particles. Also, it exhibits the spherical shape of nanoparticles whose size was found to be in between $50-20 \mathrm{~nm}$.

\subsection{Experimental investigation}

Efficient heat transfer property of homogenous MNF can be determined by Maxwell model. This model can be applicable to randomly dispersed and uniformly sized noncontacting spherical particles with base fluids [18].

$$
\frac{k_{e f f}}{k_{1}}=\frac{k_{p}+2 k_{1}+2 \phi\left(k_{p}-k_{1}\right)}{k_{p}+2 k_{1}-\phi\left(k_{p}-k_{1}\right)}
$$

where $k_{\text {eff, }} k_{l}, k_{p}$ denotes the effective, liquid and particle thermal conductivity and $\phi$ is the volume fraction of the particles. Experiments revealed the existence of heat transfer increment in magnetic fluids beyond the Maxwell limit of $3 \phi$. In the limit of low particle volume concentration $(\phi)$, the particle conductivity $\left(k_{p}\right)$ being much higher than the base liquid conductivity $\left(k_{1}\right)$, the above equation can be reduced to Maxwell $3 \phi$ limit as [18]:

$k_{\text {low }}=\frac{k_{\text {eff }}}{k_{1}}=1+3 \phi$,

where $k_{\text {low }}$ denote the lower limit of heat conduction in nanofluids. Eq. 5 represents the lower limit for the heat conduction of nanofluids in absence of particles with limit $\phi=0$, Eq. 6 represents $k_{\text {low }}$ as expected. An upper limit for heat conduction of nanofluids is established by coupling parameters such as particle size, shape, Brownian motion of particles and surfactant thickness of nanoparticles enhances the heat conduction in nanofluids. Shukla et al. reported the corresponding equation based on Brownian motion of nanoparticles in homogenous medium and Maxwell model [18]:

$k_{\text {eff }}=k_{1}\left(\frac{k_{p}+2 k_{1}+2 \phi\left(k_{p}-k_{1}\right)}{k_{p}+2 k_{1}-\phi\left(k_{p}-k_{1}\right)}+c \frac{\phi\left(T-T_{0}\right)}{\mu a^{4}}\right)$,

where $C$ is a constant whose value is $7 \times 10^{-36}, \mu$ is the dynamic viscosity, $T$ and $T_{0}$ are the variable and reference temperature and $a$ is the radius of the particle.

\subsection{Analysis of base fluids heat transfer rate}

An experimental setup shown in Fig. 1 was used to analyze the heat transfer rate of base fluids (deionized water and transformer oil) without suspended cobalt manganese nanopowders and varying magnetic field. In this experimental process, the required volume of deionized water and transformer oil were heated in $500 \mathrm{ml}$ glass beakers with a heater connected to an AC power supply of $50 \mathrm{~Hz}$ frequency and their heat transfer rate per degree with respect to time without applying magnetic field strength was noted down. It was about $4.5 \mathrm{~s} \pm 0.3$ and $4 \mathrm{~s} \pm 0.2$ respectively.

\subsection{Analysis of deionized water based magnetic fluids heat transfer rate}

Deionized water based $1.25 \%$ volume fraction of cobalt manganese nanopowder $\left(\mathrm{Co}_{1-\mathrm{x}} \mathrm{Mn}_{\mathrm{x}} \mathrm{Fe}_{2} \mathrm{O}_{4}\right)$ dispersed magnetic fluids were heated in $500 \mathrm{ml}$ glass beakers up to its maximum boiling temperature in absence of varying magnetic field. The heat transfer rate per degree with respect to time is shown in Fig. 3.

From the obtained result, the heat transfer rate of magnetic fluids $\mathrm{CoFe}_{2} \mathrm{O}_{4}$ and $\mathrm{MnFe}_{2} \mathrm{O}_{4}$ in absence of varying magnetic field strength was $2.7 \mathrm{~s} \pm 0.7$ and 
$2.8 \mathrm{~s} \pm 0.8$ respectively. The heat transfer of $\mathrm{Co}_{0.7} \mathrm{Mn}_{0.3} \mathrm{Fe}_{2} \mathrm{O}_{4}, \quad \mathrm{Co}_{0.5} \mathrm{Mn}_{0.5} \mathrm{Fe}_{2} \mathrm{O}_{4}$ and $\mathrm{Co}_{0.3} \mathrm{Mn}_{0.7} \mathrm{Fe}_{2} \mathrm{O}_{4}$ magnetic fluids was $2.6 \mathrm{~s} \pm 0.6,2.8 \mathrm{~s} \pm 0.6$ and $2.8 \mathrm{~s} \pm 0.8$ respectively.

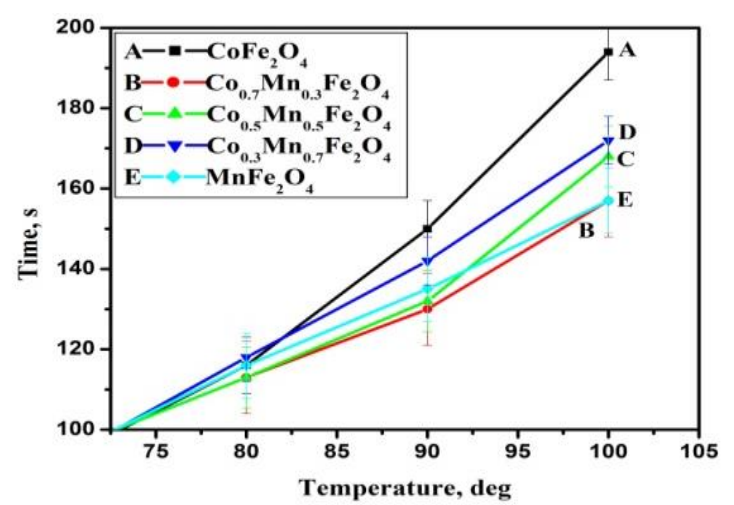

Fig. 3. The rate of heat transfer in deionized water based cobalt manganese ferrite magnetic fluids in absence of varying magnetic field

\subsection{Analysis of deionized water based magnetic fluids heat transfer rate in varying magnetic fields}

In this analysis, deionized water based $1.25 \%$ volume fraction of cobalt manganese ferrite based $\left(\mathrm{Co}_{1-\mathrm{x}} \mathrm{Mn}_{\mathrm{x}} \mathrm{Fe}_{2} \mathrm{O}_{4}\right)$ magnetic fluids placed in electromagnet arrangement were heated in varying magnetic fields for $\mathrm{I}=1.8 \mathrm{~A}$ and $2.7 \mathrm{~A}$ (current values). The varying magnetic fields were applied in perpendicular direction in accordance with the thermal gradient of magnetic fluids. The heat transfer rate per degree with respect to time is shown in Fig. 4 and Fig. 5.

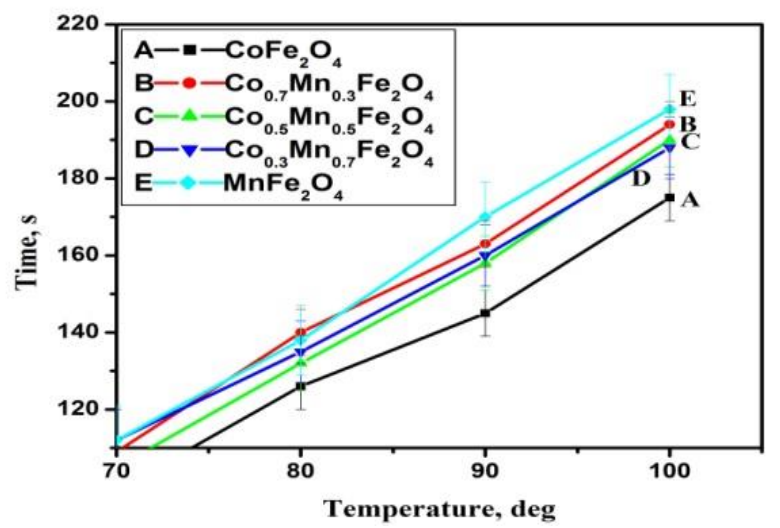

Fig. 4. The rate of heat transfer in deionized water based cobalt manganese ferrite magnetic fluids in presence of varying magnetic field for $\mathrm{I}=1.8 \mathrm{~A}$

The results specify that the heat transfer rate (for $\mathrm{I}=1.8 \mathrm{~A}$ ) per degree of $\mathrm{CoFe}_{2} \mathrm{O}_{4}$ and $\mathrm{MnFe}_{2} \mathrm{O}_{4}$ magnetic fluids was $2.9 \mathrm{~s} \pm 0.6$ and $3.3 \mathrm{~s} \pm 0.8$. The heat transfer rate (for $\mathrm{I}=1.8 \mathrm{~A}$ ) per degree of $\mathrm{Co}_{0.7} \mathrm{Mn}_{0.3} \mathrm{Fe}_{2} \mathrm{O}_{4}$, $\mathrm{Co}_{0.5} \mathrm{Mn}_{0.5} \mathrm{Fe}_{2} \mathrm{O}_{4}$ and $\mathrm{Co}_{0.3} \mathrm{Mn}_{0.7} \mathrm{Fe}_{2} \mathrm{O}_{4}$ magnetic fluids was $3.2 \mathrm{~s} \pm 0.6, \quad 3.1 \mathrm{~s} \pm 0.7$ and $3.1 \mathrm{~s} \pm 0.8$ respectively. Correspondingly, the rate of heat transfer (for $\mathrm{I}=2.7 \mathrm{~A}$ ) of $\mathrm{CoFe}_{2} \mathrm{O}_{4}$ and $\mathrm{MnFe}_{2} \mathrm{O}_{4}$ magnetic fluids was about $3.9 \mathrm{~s} \pm 0.6$ and $4.0 \mathrm{~s} \pm 0.6$. The heat transfer rate (for $\mathrm{I}=2.7 \mathrm{~A}$ ) per degree of $\mathrm{Co}_{0.7} \mathrm{Mn}_{0.3} \mathrm{Fe}_{2} \mathrm{O}_{4}, \mathrm{Co}_{0.5} \mathrm{Mn}_{0.5} \mathrm{Fe}_{2} \mathrm{O}_{4}$ and $\mathrm{Co}_{0.3} \mathrm{Mn}_{0.7} \mathrm{Fe}_{2} \mathrm{O}_{4}$ magnetic fluids was $3.2 \mathrm{~s} \pm 0.7$, $3.7 \mathrm{~s} \pm 0.4$ and $4.2 \mathrm{~s} \pm 0.5$ respectively. The average heat transfer rate of deionized water based cobalt manganese ferrite magnetic fluids $\left(\mathrm{Co}_{1-\mathrm{x}} \mathrm{Mn}_{\mathrm{x}} \mathrm{Fe}_{2} \mathrm{O}_{4}\right)$ in absence of magnetic field was about $2.7 \mathrm{~s} \pm 0.7 \mathrm{~s}$. The average heat transfer rate of magnetic fluids in presence of varying magnetic fields for $\mathrm{I}=1.8 \mathrm{~A}$ and $2.7 \mathrm{~A}$ were $3.1 \mathrm{~s} \pm 0.7$ and $3.8 \mathrm{~s} \pm 0.56$ respectively.

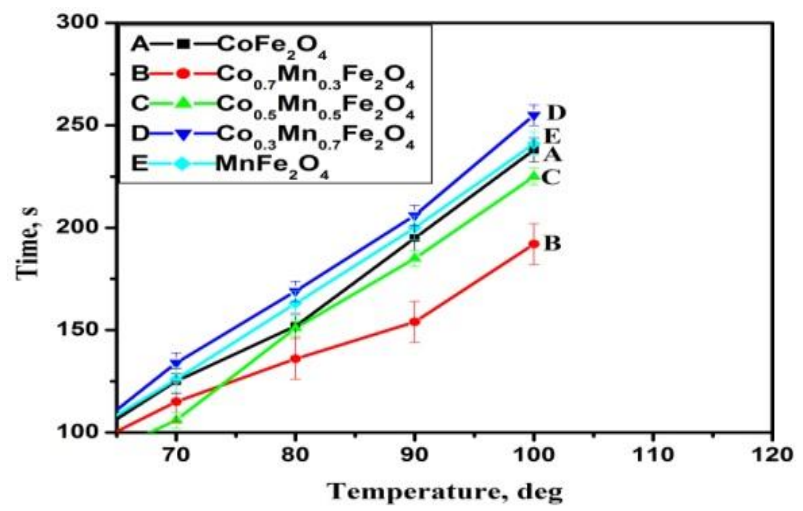

Fig. 5. The rate of heat transfer in deionized water based cobalt manganese ferrite magnetic fluids in presence of varying magnetic field for $\mathrm{I}=2.7 \mathrm{~A}$

It shows that the deionized water based cobalt manganese ferrite magnetic fluids $\left(\mathrm{Co}_{1-\mathrm{x}} \mathrm{Mn}_{\mathrm{x}} \mathrm{Fe}_{2} \mathrm{O}_{4}\right)$ shows enhancement in heat transfer rate in absence of magnetic field with more uncertainty than the magnetic fluids in presence of different magnetic fields for corresponding different current values. Here the applied varying magnetic field in perpendicular direction in accordance with the thermal gradient of magnetic fluids controls the heat transfer rate of magnetic fluids. Also, the increasing varying magnetic field strength along perpendicular direction in accordance with the thermal gradient of magnetic fluids retards the heat transfer rate. In magnetic nanoparticle suspension, each particle acts as single domain superparamagnet with a magnetic moment. The interparticle dipole-dipole interaction $U_{d}(i j)$ between the magnetic particles is [19]:

$U_{d}^{i j}=-\left[3 \frac{\left(m_{i} \cdot r_{i j}\right) \cdot\left(m_{i} \cdot r_{i j}\right)}{r_{i j}^{5}}-\frac{\left(m_{i} \cdot m_{j}\right)}{r_{i j}^{3}}\right], r_{i j}=r_{i}-r_{j}$,

where $m_{i}$ and $m_{j}$ are the magnetic moments of $i^{\text {th }}$ and $j^{\text {th }}$ particles. $r_{i}$ and $r_{j}$ are the relative positions of particles to a reference coordinate system. $r_{i j}$ is the distance of separation between $i^{\text {th }}$ and $j^{\text {th }}$ particles. The dipole interaction between the particles depends upon the distance $r_{i j}$ and with respect to the mutual orientation of magnetic moment $m_{i}$ and $m_{j}$. Hence the direction of the applied magnetic field and the magnetic moment orientation with respect to the applied direction play an important role in heat transport phenomena. Philip et al. [20] reported reduction in thermal conductivity of hexadecane based $\mathrm{Fe}_{3} \mathrm{O}_{4}$ nanofluids, when the field shifted from parallel to perpendicular direction with respect to thermal gradient. The proposed experimental result of Philip exactly coincides with the obtained experimental results. 


\subsection{Analysis of transformer oil based magnetic fluids heat transfer rate}

In this experimental part, transformer oil based $1.25 \%$ volume fraction of cobalt manganese nanopowders $\left(\mathrm{Co}_{1-\mathrm{x}} \mathrm{Mn}_{\mathrm{x}} \mathrm{Fe}_{2} \mathrm{O}_{4}\right)$ dispersed magnetic fluids were heated in $500 \mathrm{ml}$ glass beakers up to its maximum boiling temperature without applying varying magnetic field. The heat transfer rate per degree with respect to time is shown in Fig. 6.

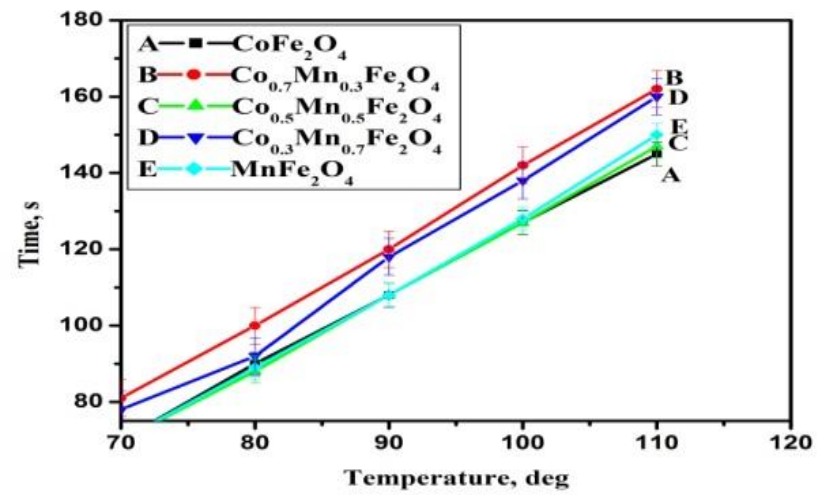

Fig. 6. The rate of heat transfer in transformer oil based cobalt manganese ferrite magnetic fluids in absence of varying magnetic field

From the observed results, the heat transfer rate of magnetic fluids $\quad \mathrm{CoFe}_{2} \mathrm{O}_{4}, \quad \mathrm{Co}_{0.7} \mathrm{Mn}_{0.3} \mathrm{Fe}_{2} \mathrm{O}_{4}$, $\mathrm{Co}_{0.5} \mathrm{Mn}_{0.5} \mathrm{Fe}_{2} \mathrm{O}_{4}, \mathrm{Co}_{0.3} \mathrm{Mn}_{0.7} \mathrm{Fe}_{2} \mathrm{O}_{4}$ and $\mathrm{MnFe}_{2} \mathrm{O}_{4}$ without applying varying magnetic field was $2.0 \mathrm{~s} \pm 0.3,2.3 \mathrm{~s} \pm 0.4$, $2.2 \mathrm{~s} \pm 0.3,2.1 \mathrm{~s} \pm 0.4$ and $2.1 \mathrm{~s} \pm 0.3$ respectively.

\subsection{Analysis of transformer oil based magnetic fluids heat transfer rate in varying magnetic field}

In this analysis, transformer oil based $1.25 \%$ volume fraction of cobalt manganese ferrite nanopowders $\left(\mathrm{Co}_{1-\mathrm{x}} \mathrm{Mn}_{\mathrm{x}} \mathrm{Fe}_{2} \mathrm{O}_{4}\right)$ dispersed magnetic fluids placed in electromagnet arrangement were heated up to its maximum boiling temperature. Simultaneously, varying magnetic field for $\mathrm{I}=1.8 \mathrm{~A}$ and $2.7 \mathrm{~A}$ (current values) was applied in perpendicular direction in accordance with thermal gradient of magnetic fluids. The heat transfer rate with respect to time is shown in Fig. 7 and Fig. 8.

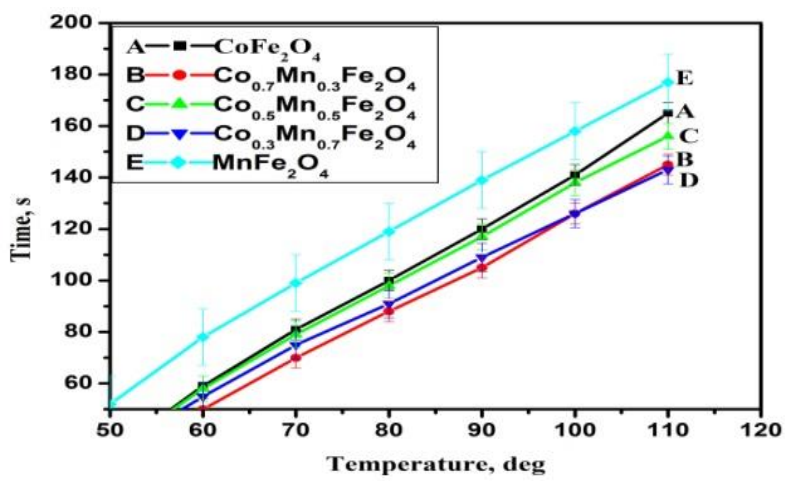

Fig. 7. The rate of heat transfer in transformer oil based cobalt manganese ferrite magnetic fluids in presence of varying magnetic field for $\mathrm{I}=1.8 \mathrm{~A}$

The results state that the heat transfer rate (for $\mathrm{I}=1.8 \mathrm{~A}$ ) per degree of $\mathrm{CoFe}_{2} \mathrm{O}_{4}$ and $\mathrm{MnFe}_{2} \mathrm{O}_{4}$ magnetic fluids was
$2.3 \mathrm{~s} \pm 0.4$ and $2.5 \mathrm{~s} \pm 0.4$. The heat transfer rate (for $\mathrm{I}=1.8 \mathrm{~A})$ per degree of $\mathrm{Co}_{0.7} \mathrm{Mn}_{0.3} \mathrm{Fe}_{2} \mathrm{O}_{4}, \mathrm{Co}_{0.5} \mathrm{Mn}_{0.5} \mathrm{Fe}_{2} \mathrm{O}_{4}$ and $\mathrm{Co}_{0.3} \mathrm{Mn}_{0.7} \mathrm{Fe}_{2} \mathrm{O}_{4}$ magnetic fluids was $2.0 \mathrm{~s} \pm 0.4$, $2.2 \mathrm{~s} \pm 0.3$ and $2.0 \mathrm{~s} \pm 0.3$ respectively. Similarly, the heat transfer rate (for $\mathrm{I}=2.7 \mathrm{~A}$ ) per degree of $\mathrm{CoFe}_{2} \mathrm{O}_{4}$ and $\mathrm{MnFe}_{2} \mathrm{O}_{4}$ magnetic fluids was $2.1 \mathrm{~s} \pm 0.4$ and $2.0 \mathrm{~s} \pm 0.4$. The heat transfer rate (for $\mathrm{I}=2.7 \mathrm{~A}$ ) per degree of $\mathrm{Co}_{0.7} \mathrm{Mn}_{0.3} \mathrm{Fe}_{2} \mathrm{O}_{4}, \quad \mathrm{Co}_{0.5} \mathrm{Mn}_{0.5} \mathrm{Fe}_{2} \mathrm{O}_{4}$ and $\mathrm{Co}_{0.3} \mathrm{Mn}_{0.7} \mathrm{Fe}_{2} \mathrm{O}_{4}$ magnetic fluids was $2.1 \mathrm{~s} \pm 0.3,2.1 \mathrm{~s} \pm 0.3$ and $2.3 \mathrm{~s} \pm 0.4$ respectively.

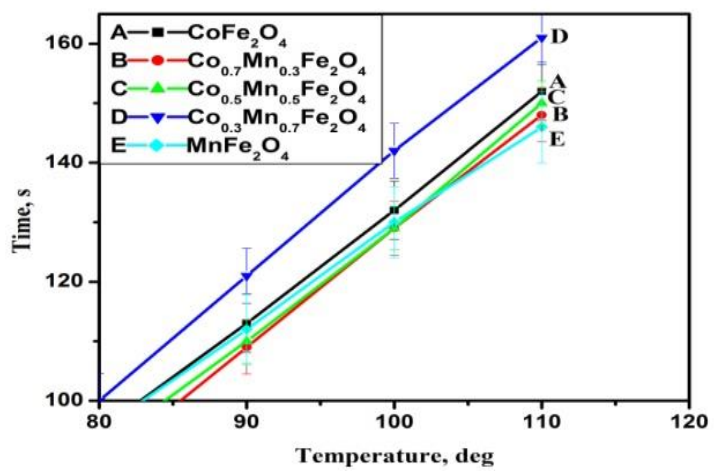

Fig. 8. The rate of heat transfer in transformer oil based cobalt manganese ferrite magnetic fluids in presence of varying magnetic field for $\mathrm{I}=2.7 \mathrm{~A}$

The results show that the rate of heat transfer in transformer oil without suspended magnetic nanoparticles was about $4 \mathrm{~s} \pm 0.2$ respectively, whereas the mean heat transfer rate of cobalt manganese ferrite based magnetic fluids $\left(\mathrm{Co}_{1-\mathrm{x}} \mathrm{Mn}_{\mathrm{x}} \mathrm{Fe}_{2} \mathrm{O}_{4}\right)$ without applying magnetic field was around $2.1 \mathrm{~s} \pm 0.34$. The results stipulate that the heat transfer rate of cobalt manganese ferrite nanopowders dispersed transformer oil (MNF) is greater than normal transformer oil. The obtained results coincide with the proposed experimental results of Philip et al. [21]. His experimental results reveal that the effective thermal conductivity in nanofluids was due to the chain like structural formation of magnetic nanoparticles dispersed in basefluids. In the same analysis, the average heat transfer rate of cobalt manganese ferrite based magnetic fluids with varying magnetic fields for $\mathrm{I}=1.8 \mathrm{~A}$ and $2.7 \mathrm{~A}$ (current values) were $2.2 \mathrm{~s} \pm 0.42$ and $2.1 \mathrm{~s} \pm 0.36$ respectively. It was observed that the uncertainty in the measurements seems to exist more for deionized water based cobalt manganese ferrite magnetic fluids than the transformer oil based cobalt manganese ferrite magnetic fluids. When comparing the obtained results, the transformer oil based cobalt manganese ferrite magnetic fluids do not show any variation in heat transfer rate with and without applying varying magnetic field. It is because; the maximum enhancement of heat transfer rate in magnetic fluids is possible only when the magnetic field is applied in parallel direction to the thermal gradient, whereas no improvement will be observed when the applied magnetic field is in perpendicular direction to the thermal gradient [22].

\subsection{Applications}

Magnetic nanofluids can be used as heat transport medium in energy convention systems, MEMS, NEMS and miniature devices $[8,19]$. Hence, $\mathrm{Co}_{1-\mathrm{x}} \mathrm{Mn}_{\mathrm{x}} \mathrm{Fe}_{2} \mathrm{O}_{4}$ fluids may 
also be used as fluids for smart cooling devices, miniature electronic devices and in transformers.

\section{CONCLUSION}

In this study, deionised water and transformer oil based cobalt manganese ferrite magnetic fluids $\left(\mathrm{Co}_{1-\mathrm{x}} \mathrm{Mn}_{\mathrm{x}} \mathrm{Fe}_{2} \mathrm{O}_{4}\right)$ coated with oleic acid were synthesized via co-precipitation technique. An experimental investigation was carried out on heat transfer rate measurements of cobalt manganese ferrite based magnetic fluids in varying magnetic field applied perpendicular in accordance with the thermal gradient of magnetic fluids. The experimental results stipulate that the heat transfer rate of cobalt manganese ferrite $\left(\mathrm{Co}_{1-\mathrm{x}} \mathrm{Mn}_{\mathrm{x}} \mathrm{Fe}_{2} \mathrm{O}_{4}\right)$ suspended base fluids show enhanced heat transfer rate than normal base fluids in absence of magnetic field. The similar magnetic fluids show decrement in heat transfer rate, when the varying magnetic fields are applied perpendicular in accordance with the thermal gradient of magnetic fluids. The result specifies that the heat transfer rate of cobalt manganese ferrite based magnetic fluids can be tunable with the direction and applied magnetic field strength. The above investigation proposes that the prepared cobalt manganese ferrite based magnetic fluids could be used as coolants in miniature electronic devices, engines and transformers.

\section{Acknowledgment}

Dr. S. Sendhilnathan gratefully acknowledges the DST (Ref. No. SERC no.100/IFD/7194/2010-11 dated 12.10.10) for the financial assistance received through the project.

\section{REFERENCES}

1. Eastman, J.A., Phillpot, S.R., Choi, S.U.S., Keblinski, P. Thermal Transport in Nanofluids Annual Review of Materials Research 34 2004: pp. 219-246. https://doi.org/10.1146/annurev.matsci.34.052803.090621

2. Eastman, J.A., Choi, U.S., Li, S., Thompson, L.J., Lee, S. Enhanced Thermal Conductivity through the Development of Nanofluids Materials Research Society Symposium Proceedings 457 1997: pp. 3 - 11.

3. Lee, S., Choi, S.U.S., Li, S., Eastman, J.A. Measuring Thermal Conductivity of Fluids Containing Oxide Nanoparticles Journal of Heat Transfer 121 1999: pp. 280-289.

4. Eastman, J.A., Choi, S.U.S., Li, S., Yu, W., Thompson, L.J. Anomalously Increased Effective Thermal Conductivities of Ethylene Glycol Based Nanofluids Containing Copper Nanoparticles Applied Physics Letters 78 (6) 2001: pp. $718-720$.

5. Xuan, Y., Li, Q. Heat Transfer Enhancement of Nanofluids International Journal of Heat and Fluid Flow 21 2000: pp. $58-64$. https://doi.org/10.1016/S0142-727X(99)00067-3

6. Xie, H., Wang, J., Xi, T., Liu, Y. Thermal Conductivity of Suspensions Containing Nanosized SiC Particles International Journal of Thermophysics 23 (2) 2002: pp. 571-580. https://doi.org/10.1023/A:1015121805842
7. Kurikiyimfura, I., Wang, Y., Pan, Z. Heat Transfer Enhancement by Magnetic Nanofluids - A Review Renewable and Sustainable Energy Reviews 21 2013: pp. 548-561.

8. Shima, P.D., Philip, J., Raj, B. Magnetically Controllable Nanofluid with Tunable Thermal Conductivity and Viscosity Applied Physics Letters 95 2009: pp. 133112-1-13112-3.

9. Vaidyanathan, G., Sendhilnathan, S., Arulmurugan, R. Structural and Magnetic Properties of $\mathrm{Co}_{1-x} \mathrm{Zn}_{\times} \mathrm{Fe}_{2} \mathrm{O}_{4}$ Nanoparticles by Co-precipitation Method Journal of Magnetism and Magnetic Materials 313 2007: pp. 293-299.

10. Vaidyanathan, G., Sendhilnathan, S. Synthesis and Magnetic Properties of Co-Zn Magnetic Fluid Journal of Magnetism and Magnetic Materials 320 (6) 2008: pp. 803-805. https://doi.org/10.1016/j.jmmm.2007.08.021

11. Philip, J., Shima, P.D., Raj, B. Evidence for Enhanced Thermal Conduction through Percolating Structures Nanotechnology 19 (30) 2008: pp. 305706-1-305706-7.

12. Gavili, A., Zabihi, F., Isfahani, T.D., Sabbaghzadeh, J. The Thermal Conductivity of water Base Ferrofluids under Magnetic field Experimental Thermal and Fluid Science 41 2012: pp. $94-98$. https://doi.org/10.1016/j.expthermflusci.2012.03.016

13. Li, D., Jiang, D., Chen, M., Xie, J., Wu, Y., Dang, S., Zhang, J. An Easy Fabrication of Monodisperse Oleic AcidCoated $\mathrm{Fe}_{3} \mathrm{O}_{4}$ Nanoparticles Materials Letters 64 2010: pp. $2462-2464$. https://doi.org/10.1016/j.matlet.2010.08.025

14. Krichler, M., Odenbach, $\mathbf{S}$. Thermal Conductivity Measurements on Ferrofluids with Special Reference to Measuring Arrangement Journal of Magnetism and Magnetic Materials 326 2013: pp. 85-90.

15. Thurm, S., Odenbach, S. Particle Size Distribution as Key Parameter for the Flow Behavior of Ferrofluids Physics of Fluids 15 (6) 2003: pp. $1658-1664$.

16. Yadav, S.P., Shinde, S.S., Bhatt, P., Meena, S.S., Rajpure, K.Y. Distribution of Cations in $\left(\mathrm{Co}_{1-\mathrm{x}} \mathrm{Mn}_{\mathrm{x}} \mathrm{Fe}_{2} \mathrm{O}_{4}\right)$ Using XRD, Magnetization and Mossbauer Spectroscopy Journal of Alloys and Compounds 646 2015: pp. 550-556. https://doi.org/10.1016/j.jallcom.2015.05.270

17. Ashiq, M.N., Saleem, S., Malana, M.A., Rehman, A.U., Physical Electrical and Magnetic Properties of Nanocrystalline $\mathrm{Zr}$-Ni Doped Mn-ferrite Synthesized by The Co-Precipitation Method Journal of Alloys and Compounds 486 2009: pp. $640-644$.

18. Murugesan, C., Sivan, S. Limits for Thermal Conductivity of Nanofluids Thermal science 14 2010: pp. 65-71.

19. Kurikiyimfura, I., Wang, Y., Pan, Z., Hu, D. Thermal Conductivity Enhancement of Magnetic Nanofluid in Magnetic Field International Conference on Materials for Renewable Energy \& Environment (ICMREE) 2 2011: pp. 1333-1337.

20. Shima, P.D., Philip, J. Tuning of Thermal Conductivity and Rheology of Nanofluids Using an External Stimulus The Journal of Physical Chemistry C 115 2011: pp. 20097-20104.

21. Philip, J., Shima, P.D., Raj, B. Enhancement of Thermal Conductivity in Magnetite Based Nanofluid Due to Chainlike Structures Applied Physics Letters 91 (20) 2007: pp. 2031081-203108-3.

22. Lian, W., Xuan, Y., Li, Q. Characterization of Miniature Automatic Energy Transport Devices Based on the Thermomagnetic Effect Energy Conversion and Management 50 2009: pp. $35-42$. 